\title{
Concept Paper \\ Drug Repositioning Ketamine as a New Treatment for Bipolar Disorder Using Text Mining
}

\author{
Shivani Manikandan ${ }^{1, *}$, Suchir Misra ${ }^{2,3, *}$ and Serena McCalla ${ }^{2, *}$ \\ 1 Princeton Day School, Princeton, NJ 08540, USA \\ 2 iResearch Institute, Glen Cove, NY 11542, USA \\ 3 Faculty of BioSciences, Rice University, Huston, TX 77005, USA \\ * Correspondence: shivani.manikandan@icloud.com (S.M.); smisra@iresearchinstitute.com (S.M.); \\ admin@iresearchinstitute.com (S.M.)
}

Citation: Manikandan, S.; Misra, S.; McCalla, S. Drug Repositioning Ketamine as a New Treatment for Bipolar Disorder Using Text Mining. BioChem 2022, 2, 1-7. https:// doi.org/10.3390/biochem2010001

Academic Editor: Alessandro Alaimo

Received: 24 October 2021

Accepted: 24 December 2021

Published: 31 December 2021

Publisher's Note: MDPI stays neutral with regard to jurisdictional claims in published maps and institutional affiliations.

Copyright: (c) 2021 by the authors Licensee MDPI, Basel, Switzerland. This article is an open access article distributed under the terms and conditions of the Creative Commons Attribution (CC BY) license (https:// creativecommons.org/licenses/by/ $4.0 /)$.

\begin{abstract}
Bipolar Disorder (BD), a chronic mental illness, does not have an ideal treatment, and patients with BD have a higher chance of being diagnosed with alcohol abuse, liver disease, and diabetes. The goal of treatment is to prevent a relapse in BD episodes and find a new treatment. The research here looks at the genetics of $\mathrm{BD}$ and ignores environmental factors, as they are subjective. Therapy treats known environmental triggers and stressors and explores methods to reduce them. However, therapy alone cannot fully alleviate the symptoms of BD. My research employs text-mining as a primary strategy to obtain relevant genes and drugs pertaining to BD. The main gene involved is the Brain-Derived Neurotrophic Factor (BDNF). Popular drugs currently used for treatment of BD are Lithium and Carbamazepine. Using CMapPy to look at gene expression data, one sees a relationship between the two drug therapies and BDNF. Lithium fails to treat mania and Carbamazepine fails to treat depression, relatively speaking. When comparing gene expression data of Lithium and Carbamazepine with Ketamine, a newer therapy for BD, Ketamine, raises the BDNF level, keeps it elevated, and effectively controls BD episodes. Ketamine does not have the shortcomings that Lithium and Carbamazepine have. Next steps would include conducting a clinical trial with the hopeful application of Ketamine as a new treatment for BD.
\end{abstract}

Keywords: bipolar disorder; drug repositioning; text-mining; Wnt; BDNF; genetics; ketamine; lithium; carbamazepine

\section{Introduction}

Bipolar disorder, which affects 1 in 100 people, does not currently have any therapies that prevent relapses in patients' symptoms [1-3]. BD swings a person's mood as a person enters a depressive episode, a state in which one becomes lethargic and can be suicidal, unable to get out of bed to do something, to a manic episode, where one acts impulsively and unwillingly moves constantly, with time in between episodes. This inconsistent, uncontrollable lifestyle needs proper treatment to alleviate the stress, pain, and to prevent relapses. Current treatments do not prevent new episodes from occurring, and existing therapies cannot identify new triggers. Additionally, BD patients tend to abuse alcohol, which can lead to increased incidents of liver disease, often present in people with BD [4-6]. These diseases can worsen the severity of the episodes and could lead to further complications.

The Wingless-related Integration Site (Wnt) is the pathway involved that causes episodes $[7,8]$. Dysregulation of the Wnt signaling pathway is caused by a mutation in the Brain Derived Neurotrophic Factor (BDNF) gene [9]. Due to the mutation, as the pathway moves forward, the b-catenin protein becomes downregulated, leading to downregulated gene transcription in the DNA, causing the antidepressant effect (when someone goes into a manic or depressive state-one of their episodes) (Figure 1). 


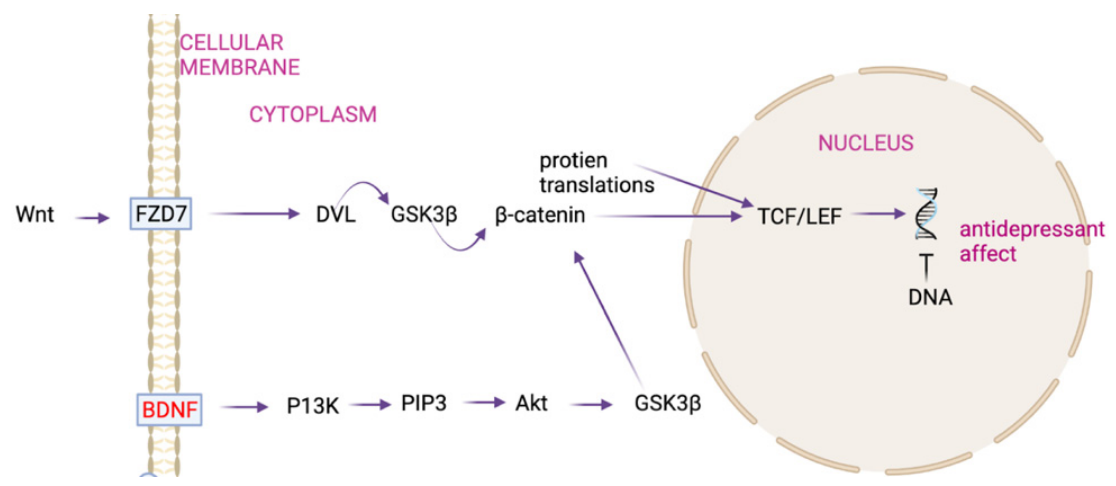

Figure 1. Diagram of the Wingless-related integration site as it functions in a person with Bipolar Disorder. The genes mentioned are Frizzled homolog 7 (FZD7), Glycogen Synthase Kinase 3 Beta (GSK3B), Beta Catenin (B-catenin), T-cell factor/lymphoid enhancer factor (TCF/LEF), Brain-Derived Neurotrophic Factor (BDNF), Phosphoinositide 3-kinases (P13K), phosphoinositide 3 kinase (PIP3), Protein kinase B (Akt). The diagram illustrates how, starting with the BDNF gene, the pathway functions, eventually leading to the antidepressant effect in people.

There are some major flaws with current drug treatments (Table 1). Although they treat some aspects of BD, drugs do not treat all aspects. The drug predominantly used, Lithium, does not target manic episodes nor rapid cycling (switching from manic to depressive episodes without a gap). Carbamazepine, despite targeting the manic episodes, also does not alleviate rapid cycling. In addition, drugs do not always work with depressive states despite being the therapy's target.

Table 1. Drugs currently used to treat Bipolar Disorder are classified to look at their functions, flaws, effectiveness, etc. This was completed before receiving data from the text-mining and organizing them (Table 2), in which the drugs found were based on manual research in PubMed.

\begin{tabular}{|c|c|c|}
\hline Name of Drug & Flaws of Drug & $\begin{array}{l}\text { Effectiveness with } \\
\text { Depressive Episodes }\end{array}$ \\
\hline Lithium & $\begin{array}{l}\text { Lots of bloodwork, unknown } \\
\text { consequences, unpleasant side effects, } \\
\text { can cause diabetes, weight gain }\end{array}$ & Works well \\
\hline Olanzapine & $\begin{array}{l}\text { Difficulty walking, seizures, weight } \\
\text { gain, unusual behavior }\end{array}$ & Works moderately well \\
\hline Quetiapine & $\begin{array}{l}\text { Pain, loss of coordination, unusual } \\
\text { dreams, excessive weight gain, } \\
\text { difficulty speaking/concentrating }\end{array}$ & Works well \\
\hline Risperidone & $\begin{array}{l}\text { Vision issues, anxiety, weight gain, } \\
\text { stomach pain, fever, difficulty } \\
\text { breathing, irregular pulse }\end{array}$ & Works well \\
\hline Carbamazepine & Dizziness, balance issues & Works moderately well \\
\hline Aripiprazole & $\begin{array}{l}\text { Pain, tiredness, nervousness, } \\
\text { dizziness, seizures, tightening } \\
\text { of muscles }\end{array}$ & Works well \\
\hline
\end{tabular}

Drugs also have side effects (e.g., seizures, memory loss, ambulatory and balance issues, etc.) which can lead to worsened BD episodes or symptoms. Therapy treatments, despite their efforts to give patients coping mechanisms and ways to deal with stress, fail to identify any new triggers. They only focus on known triggers and ways to calm down patients. Clearly, the commonly used drug treatments are not effective, despite being a common disorder. 
Table 2. Number of times specific genes have been represented in relationship with Bipolar Disorder. After receiving data from the code, the data were organized, and then validated.

\begin{tabular}{cc}
\hline Gene Name & Occurrence \\
\hline BDNF & 224 \\
COMT & 136 \\
CACNA1C & 130 \\
DISC1 & 112 \\
ANK3 & 86 \\
DRD2 & 63 \\
NRG1 & 57 \\
DRD4 & 56 \\
SLC6A4 & 49 \\
CLOCK & 45 \\
\hline
\end{tabular}

\section{Materials and Methods}

\subsection{Text Mining for Genes and Drugs}

Current drug therapies only control symptoms of Bipolar Disorder and do not affect the actual genes that bring about BD in the first place. Both the genes that cause BD and the drug therapies that treat it need to be catalogued. To extract the information needed from PubMed, text-mining code was written in Python and divided into two routines; one generates a list of genes involved with $\mathrm{BD}$ and the other generates a list of drugs that have been researched as treatment options for BD. The process worked thus: using Python, the text-mining code created two separate routines. The first routine generated a list of abstract IDs (e.g., 34249777, 34207921, 34207460). For genes, the search term 'Bipolar Disorder Genes' was used and for drugs, the search term 'Bipolar Disorder Drugs' was used.

The second routine extracted information. A given list (in text file format) was used as a reference to all necessary genes and drugs that are worthwhile to look at. This was taken from the HGNB and DGIDb. In doing so, two databases became references and the program searches for all those terms within the abstracts. In each abstract in which text mining was conducted, the punctuation was removed such that each word was its own separate entity, then searched for any of the terms in the given database (the genes for the gene program and the drugs for the drug program). The output was written to a separate text file. After organizing the data received, the most significant genes and drugs were obtained. These were validated by reading various literature regarding the genes. Since only the top 10 genes needed to be received, literature was read to ensure that the specific gene was directly related to Bipolar Disorder, in a way that affects the disease.

Libraries used to put together the programs were the pandas, NumPy, metapub, and Eutils libraries. Using the MetaPub library, the PubMedFetcher was imported and a variable was set to hold both the search query and the number of search results to be analyzed; in this case, 1,000,000 results were to be analyzed for the queries "bipolar disorder drugs" and "bipolar disorder genes." This allowed the code to analyze the material from the abstracts (which was separated earlier by obtaining the abstract IDs) using the metapub library. Then, it was written to a new file in which it compiled all the genes or drugs that have been mentioned. These results were then sorted using Excel.

If other scientists were to conduct similar experiments, they would have almost identical results, with varying factors including search terms, and more varying results looked at. Because of this and other factors, the specific way the code is written can vary, in addition to there being slightly different ways to achieve the same goal.

\subsection{Using CMapPy to Obtain Gene Expression Data}

In order to find new treatment, gene expression data were used to compare how drugs affect a specific gene. After putting all the data together, the drug expressing the gene that is highest for a BD patient would become the selected drug for a new treatment. 
After getting information from the previous programs and organizing and validating the data, the next steps were to relate the genes and the drugs and gene expression data. Using the data, one can look at current treatments along with possible new ones to identify the best possible treatment options for BD patients.

CMapPy is a library that allows Python programmers to look at specific file types and obtain data from them. By using this library, it is possible to compare data to determine which drugs would work best to treat the significant gene involved, in this case, BDNF. Using online tutorials from GitHub, it is possible to compile a list of necessary files and look at the data one has. As mentioned earlier, code will vary, and it is imperative that one has the files and follows tutorials as needed.

Parsing through the CMapPy files resulted in obtaining the effects on the genes associated with Bipolar Disorder. To find a new, acceptable treatment, gene expression data for both current and possible treatments allowed us to compare them to select the best.

\section{Results}

\subsection{Genes That Cause Bipolar Disorder}

After running the first program to obtain the list of Abstract IDs and running the second program, the list of genes was compiled. Then, after organizing the data, the top ten genes, after validation, were the genes most associated with BD. The Brain-Derived Neurotrophic Factor was the most common one that was shown, followed by the CatecholO-Methyltransferase (Table 2).

As shown by Table 2, BDNF is the gene most commonly referenced in relation to Bipolar Disorder.

\subsection{Drugs Currently Used to Treat Bipolar Disorder}

After organizing results from the text-mining genes, the same process was completed for the drugs; however, the computing code was altered slightly to fit new parameters. After organizing all the data once both programs were complete, the top ten drugs were chosen and validated as the most common current drug treatments to look at. Lithium was by far the most common drug used in treatment, followed by Carbamazepine (Table 3).

Table 3. The number of times specific drugs have been represented in relationship with Bipolar Disorder. After receiving data from the code, the data were organized, and then validated as treatment options currently used.

\begin{tabular}{cc}
\hline Drug Name & Occurrence \\
\hline Lithium & 1442 \\
Carbamazepine & 488 \\
Quetiapine & 347 \\
Lamotrigine & 342 \\
Risperidone & 267 \\
Aripiprazole & 220 \\
Clozapine & 169 \\
Haloperidol & 152 \\
Ziprasidone & 111 \\
Topiramate & 93 \\
\hline
\end{tabular}

As shown by Table 3, Lithium and Carbamazepine are the drugs most commonly referenced drugs in relation to Bipolar Disorder.

\subsection{Which New Drug Would Work Best}

Once the data were organized, the gene expression data helped to identify a drug therapy that would result in optimal treatment. The table shows how well the drugs are based on the gene expression data, allowing identification of a drug that would work best after validation, which was found to be Ketamine (Table 4). 
Table 4. Effectiveness of drug treatments based on Gene Expression Data. Data received from CMapPy and validated with research. BDNF is the Brain-Derived Neurotrophic Factor.

\begin{tabular}{ccc}
\hline Name of Drug & $\begin{array}{c}\text { Effectiveness to Treat Bipolar Based } \\
\text { on Gene Expression (BDNF) }\end{array}$ & Magnitude on Scale of 1-10 \\
\hline Lithium & $\begin{array}{c}\text { Raises BDNF, but there are some flaws } \\
\text { Simply attempts to prevent abnormal } \\
\text { levels of BDNF, there are flaws } \\
\text { Karbamazepine }\end{array}$ & 6 \\
Ketamine & Raises BDNF, research seems promising & 5 \\
\hline
\end{tabular}

When looking at the gene expression data, they were then expressed in terms magnitude, on scale 1-10, with Ketamine having the largest magnitude, showing that it would express the BDNF gene the greatest.

\section{Discussion}

Genes such as Dopamine Receptor D2 and Dopamine Receptor D4 are involved in chemicals and other parts that affect Bipolar Disorder. The Brain-Derived Neurotrophic Factor (BDNF) is the most common gene mentioned (Table 2). It helps with the survival and growth of neurons, neuroplasticity, communication, and memory [10,11]. BDNF affects Bipolar patients as there is a negative correlation between the severity of the episodes and the levels of BDNF $[9,12]$. The correlation expresses the key reason as to why the BDNF gene needs to be looked at. The takeaway from these first results is that the gene to be looking at for the remainder of the research is the BDNF gene.

Although Lithium is the chief drug used in modern-day treatment for BD, the drug has serious flaws as there is not much known about it. In addition, Lithium, despite being able to treat depression and acute mania, fails to treat intense mania and rapid cycling [13]. Additionally, taking Lithium everyday can lead to Lithium poisoning [14].

Looking at the second item on the list, Carbamazepine, it also has shortcomings as a treatment for BD. Carbamazepine does not work well with rapid cycling and does not target all parts of Bipolar [15]. The takeaway here is to look at Carbamazepine and Lithium, the genes that came up the most, and to relate them to BDNF.

Lithium and Carbamazepine are drugs that raise BDNF levels but fail to keep them constant. For Lithium, there are flaws as it does not control all aspects of BD—only the depressive and some manic symptoms. With Carbamazepine, it does not really raise the levels, it only prevents abnormal levels. In conclusion, both drugs work, but they do not seem to work as well as Ketamine would. In addition, new research supports the idea of having Ketamine as a treatment option for BD. Using the numbers received, the numbers were fitted to a scale of 1-10 so that it would be easier to understand and make sense of to determine which drug works best. The drugs looked at in this scenario are the ones most used for Bipolar treatment, as found by reading literature and using the found results from the text mining.

\section{Conclusions}

Based on data, Ketamine appears to be a more promising therapy, as it overcomes the shortcomings with previous treatments which fail to target all aspects of Bipolar Disorder and side effects leading to worsened episodes in certain cases.

Ketamine does not have any of the issues that other therapies present, despite their side effects of nausea. Ketamine raises BDNF levels and keeps them elevated, limiting any further manic or depressive episodes (Figure 2). 


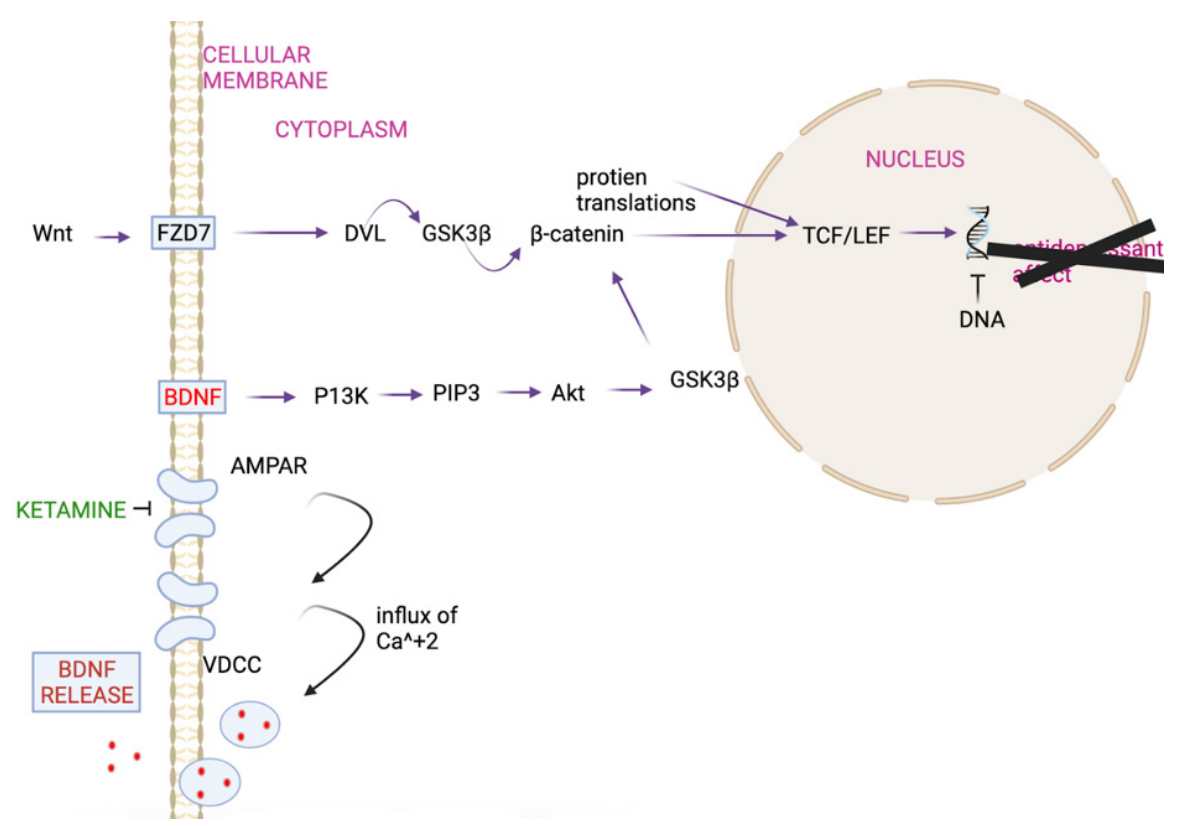

Figure 2. Diagram of the Wingless-related integration site as it functions in a person with Bipolar Disorder with Ketamine as a treatment. The mentioned genes and proteins are: Frizzled homolog 7 (FZD7), Glycogen Synthase Kinase 3 Beta (GSK3B), Beta Catenin (B-catenin), T-cell factor/lymphoid enhancer factor (TCF/LEF), Brain-Derived Neurotrophic Factor (BDNF), Phosphoinositide 3-kinases (P13K), phosphoinositide 3 kinase (PIP3), Protein kinase B (Akt), AMPAR( $\alpha$-amino-3-hydroxy-5methyl-4-isoxazolepropionic acid receptor), and VDCC (Voltage-gated calcium channel).

As shown in Figure 2, Ketamine would bring the BDNF levels back to normal, preventing the mutation there, leading to a more normal B-catenin, preventing the antidepressant effect, and limiting chances of a relapse. Looking at the magnitude by which Ketamine raises BDNF, it is significantly higher than those of Lithium and Carbamazepine, which raise BDNF levels more as a by-product as they focus on abnormal activities in the brain (Table 1.) Ketamine raises BDNF levels by taking advantage of the resting-state functional connectivity of the prefrontal cortex [16]. This is also closely related to the plasma levels, which end up raising the gene levels. By raising the levels, an increase in the production of BDNF occurs, as seen in Figure 2.

A future extension would be running a clinical trial on Bipolar Disorder patients to see the further uses of Ketamine and how it can be leveraged in treatment, allowing for new treatment applications as the idea is formed further and tested.

Author Contributions: Conceptualization, S.M. (Shivani Manikandan) and S.M. (Suchir Misra); methodology, S.M. (Shivani Manikandan) and S.M. (Suchir Misra); software, S.M. (Shivani Manikandan); validation, S.M. (Shivani Manikandan) and S.M. (Suchir Misra); formal analysis, S.M. (Shivani Manikandan); investigation, S.M. (Shivani Manikandan); resources, S.M. (Shivani Manikandan); data curation, S.M. (Shivani Manikandan); writing—original draft preparation, S.M. (Shivani Manikandan); writing-review and editing, S.M. (Shivani Manikandan) and S.M. (Suchir Misra); visualization, S.M. (Shivani Manikandan); supervision, S.M. (Suchir Misra) and S.M. (Serena McCalla); project administration, S.M. (Serena McCalla) All authors have read and agreed to the published version of the manuscript.

Funding: This project received no external funding.

Institutional Review Board Statement: Not applicable.

Informed Consent Statement: Not applicable.

Conflicts of Interest: The authors declare no conflict of interest. 


\section{References}

1. Bledsoe, A. Conditions Linked to Bipolar Disorder-Bipolar Disorder Center-Everyday Health. EverydayHealth.com. Available online: https:/ / www.everydayhealth.com/hs/bipolar-depression/bipolar-disorder-comorbid-conditions/ (accessed on 15 December 2021).

2. Overview-Bipolar Disorder. 11 February 2021. Available online: https://www.nhs.uk/mental-health/conditions/bipolardisorder/overview/ (accessed on 1 August 2021).

3. Fagiolini, A.; Chengappa, K.N.R.; Soreca, I.; Chang, J. Bipolar Disorder and the Metabolic Syndrome: Causal Factors, Psychiatric Outcomes and Economic Burden. CNS Drugs 2008, 22, 655-669. [CrossRef] [PubMed]

4. Bipolar Disorder and Alcoholism. Available online: https://pubs.niaaa.nih.gov/publications/arh26-2/103-108.htm (accessed on 1 August 2021).

5. Alcohol-Related Liver Disease. 20 October 2017. Available online: https://www.nhs.uk/conditions/alcohol-related-liverdisease-arld/ (accessed on 1 August 2021).

6. Hsu, J.-H.; Chien, I.-C.; Lin, C.-H. Increased risk of chronic liver disease in patients with bipolar disorder: A population-based study. Gen. Hosp. Psychiatry 2016, 42, 54-59. [CrossRef] [PubMed]

7. Causes-Bipolar Disorder. 11 February 2021. Available online: https://www.nhs.uk/mental-health/conditions/bipolardisorder/causes / (accessed on 1 August 2021).

8. Gould, T.D.; Manji, H.K. The Wnt Signaling Pathway in Bipolar Disorder. Neuroscientist 2002, 8, 497-511. [CrossRef] [PubMed]

9. Grande, I.; Fries, G.R.; Kunz, M.; Kapczinski, F. The Role of BDNF as a Mediator of Neuroplasticity in Bipolar Disorder. Psychiatry Investig. 2010, 7, 243. [CrossRef] [PubMed]

10. BDNF Gene: MedlinePlus Genetics. Available online: https://medlineplus.gov/genetics/gene/bdnf/ (accessed on 1 August 2021).

11. Miranda, M.; Morici, J.F.; Zanoni, M.B.; Bekinschtein, P. Brain-Derived Neurotrophic Factor: A Key Molecule for Memory in the Healthy and the Pathological Brain. Front. Cell. Neurosci. 2019, 13, 363. [CrossRef] [PubMed]

12. Sjahrir, M.; Roesyanto-Mahadi, I.D.; Effendy, E. Correlation between Serum Brain-Derived Neurotrophic Factor Level and Depression Severity in Psoriasis Vulgaris Patients. Open Access Maced. J. Med. Sci. 2019, 7, 583-586. [CrossRef] [PubMed]

13. Machado-Vieira, R.; Manji, H.K.; Jr, C.A.Z. The role of lithium in the treatment of bipolar disorder: Convergent evidence for neurotrophic effects as a unifying hypothesis. Bipolar Disord. 2009, 11 (Suppl. 2), 92-109. [CrossRef] [PubMed]

14. Purse, M. Lithium Toxicity: Types, Symptoms, Diagnosis, and Treatment-Verywell Mind. Available online: https://www. verywellmind.com/lithium-toxicity-types-causes-and-treatment-380283 (accessed on 1 August 2021).

15. Weisler, R.H. Carbamazepine extended-release capsules in bipolar disorder. Neuropsychiatr. Dis. Treat. 2006, 2, 3-11. [PubMed]

16. Ketamine, R. Ketamine's Impact on Brain-Derived Neurotrophic Factor (BDNF). Reset Ketamine-Ketamine Infusion Clinic in Palm Springs, California. Retrieved on 8 November 2021. Available online: https://www.resetketamine.com/blog/2019/3/1 7ketamines-impact-on-brain-derived-neurotrophic-factor-bdnf (accessed on 31 October 2021). 